KYUNGPOOK Math. J. 53(2013), 185-189

http://dx.doi.org/10.5666/KMJ.2013.53.2.185

\title{
A Class of Invertible Bilateral Weighted Shifts
}

\section{IL Bong JunG*}

Department of Mathematics, Kyungpook National University, Daegu, 702-701, Korea

e-mail : ibjung@knu.ac.kr

\section{Carl Pearcy}

Department of Mathematics, Texas A\&M University, College Station, TX 77843, USA

e-mail : pearcy@math.tamu.edu

ABstract. In this note we study a class of invertible weighted bilateral shifts on Hilbert space introduced by Haskell Rosenthal recently. We show that every Rosenthal shift is unitarily equivalent to its inverse, not quasisimilar to its adjoint, and has a nontrivial hyperinvariant subspace.

We write, as usual, $\mathbb{Z}$ for the set of integers and $\mathbb{N}\left(\mathbb{N}_{0}\right)$ for the set of positive (nonnegative) integers. We also write $l_{2}(\mathbb{Z})$ for the separable, infinite dimensional, complex Hilbert space

$$
l_{2}(\mathbb{Z}):=\left\{\left\{\zeta_{n}\right\}_{n \in \mathbb{Z}}: \zeta_{n} \in \mathbb{C}, \sum_{n \in \mathbb{Z}}\left|\zeta_{n}\right|^{2}<+\infty\right\}
$$

with its canonical inner product and norm. We denote, as usual, the algebra of all (bounded, linear) operators on $l_{2}(\mathbb{Z})$ by $\mathcal{L}\left(l_{2}(\mathbb{Z})\right)$. Let $\left\{e_{n}\right\}_{n \in \mathbb{Z}}$ be the canonical orthonormal basis for $l_{2}(\mathbb{Z})$. By definition, if $w=\left\{w_{n}\right\}_{n \in \mathbb{Z}}$ is any bounded sequence of positive numbers such that $\inf _{\mathbb{Z}} w_{n}>0$, then the operator $B_{w} \in \mathcal{L}\left(l_{2}(\mathbb{Z})\right)$ defined by

$$
B_{w} e_{n}=w_{n} e_{n+1}, \quad n \in \mathbb{Z}
$$

is a (invertible, forward) weighted bilateral shift. A trivial calculation gives the defining equations

$$
B_{w}^{*} e_{n}=w_{n-1} e_{n-1}, \quad n \in \mathbb{Z}
$$

* Corresponding Author.

Received January 7, 2013; accepted February 5, 2013.

2010 Mathematics Subject Classification: 47A15, 47B37.

Key words and phrases: bilateral shift, Rosenthal shift, hyperinvariant subspace. 
and

$$
B_{w}^{-1} e_{n+1}=w_{n}^{-1} e_{n}, \quad n \in \mathbb{Z} .
$$

For the reader's convenience, facts that will be needed about this class of operators will be referenced with respect to [6], where extensive remarks are made as to the origin and authorship of these results. Associated with any such shift $B_{w}$ is another sequence $\left\{\beta_{n}\right\}_{n \in \mathbb{Z}}$ of "weights" which is often found useful in the study of such operators (cf. [6]), defined by

$$
\beta_{0}=1, \beta_{n}=w_{0} \cdots w_{n-1}, \quad \beta_{-n}=\left(w_{-1} \cdots w_{-n}\right)^{-1}, \quad n \in \mathbb{N},
$$

which immediately gives

$$
w_{n}=\beta_{n+1} / \beta_{n}, \quad n \in \mathbb{Z} .
$$

Note that (4) and (5) give one the option of defining an invertible $B_{w}$ by specifying either the sequence $\left\{w_{n}\right\}_{n \in \mathbb{Z}}$ or the appropriate sequence $\left\{\beta_{n}\right\}_{n \in \mathbb{Z}}$.

A year or so ago Haskell Rosenthal introduced a particularly interesting class of these bilateral shifts, which we now define and make some general observations about (Propositions 1.2, 1.5, and Theorems 1.3, 1.4).

Definition 1.1. Let $\left\{r_{n}\right\}_{n \in \mathbb{N}}$ be any sequence of positive numbers such that

$$
r_{n} \geq 1, n \in \mathbb{N}, \quad \lim _{n \rightarrow+\infty} r_{n}=1, \text { and } \lim _{n \rightarrow+\infty} r_{n}^{n}=+\infty .
$$

Then the corresponding $B_{\left\{w_{n}\right\}}$ defined by setting

$$
\beta_{0}=1, \beta_{-n}=\beta_{n}, \beta_{n^{2}}=1, \beta_{n^{2}+j}=r_{n}^{j}, \beta_{n^{2}+n+j}=r_{n}^{n+1-j}, 1 \leq j \leq n,
$$

will henceforth herein be called a Rosenthal shift. (Note that $\inf _{n \in \mathbb{Z}} \beta_{n}=1$ for every such shift.) For the reader's convenience, we tabulate the first few $w_{n}$ associated with this sequence $\left\{\beta_{n}\right\}$. The square encloses $w_{0}$ :

$$
\begin{gathered}
\ldots, r_{3}^{-1}, r_{2}, r_{2}, 1, r_{2}^{-1}, r_{2}^{-1}, r_{1}, 1, r_{1}^{-1}, 1,1, r_{1}, 1, r_{1}^{-1}, \\
r_{2}, r_{2}, 1, r_{2}^{-1}, r_{2}^{-1}, r_{3}, r_{3}, r_{3}, 1, r_{3}^{-1}, \ldots
\end{gathered}
$$

Note that the $w_{n}$ for $n<0$ are essentially the inverses of the weights $w_{n}$ for $n \geq 0$. The exact formula for a Rosenthal shift, calculated via (4), (5), and (7) is

$$
w_{-n}=w_{n-1}^{-1}, \quad n \in \mathbb{Z} .
$$

Our first result is no suprise. 
Proposition 1.2. Every Rosenthal shift is a perturbation, by a compact bilateral shift, of the unitary, unweighted, bilateral shift, and also has its spectrum and essential (i.e., Calkin) spectrum equal to the unit circle.

Proof. Since $\lim _{|n| \rightarrow+\infty} r_{n}=1$, the first statement is obvious, and therefore the essential spectrum of the Rosenthal shift must be the unit circle. By the Fredholm theory, the spectrum must be the unit circle together with a countable number of Fredholm points. But since the spectrum of an invertible bilateral shift is known to be an annulus (cf. [6]), the only possibility for the spectrum is to be the unit circle.

Theorem 1.3. Every Rosenthal shift is unitarily equivalent to its inverse.

Proof. Observe first that two (forward, bilateral) shifts which satisfy the same defining equation (i.e., have the same weight sequences $\left\{w_{n}\right\}$ relative to some two orthonormal bases) are obviously unitarily equivalent. Now let $B_{w=\left\{w_{n}\right\}_{n \in \mathbb{Z}}}$ be any Rosenthal shift. From (3), by using (9), we obtain

$$
B_{w}^{-1} e_{n+1}=w_{-(n+1)} e_{n}, n \in \mathbb{Z} .
$$

Making a permutation of the basis $\left\{e_{n}\right\}_{n \in \mathbb{Z}}$ by defining $f_{n}=e_{-n}, n \in \mathbb{Z}$, we obtain from (10) that

$$
B_{w}^{-1} f_{-(n+1)}=w_{-(n+1)} f_{-n}, n \in \mathbb{Z},
$$

and setting $k=-(n+1)$ one gets

$$
B_{w}^{-1} f_{k}=w_{k} f_{k+1}, k \in \mathbb{Z},
$$

which is the same equation as (1) and completes the proof.

Unfortunately this nice relation between a Rosenthal shift and its inverse does not carry over to a relation with its adjoint.

Theorem 1.4. No Rosenthal shift is quasisimilar to its adjoint.

Proof. It is a result of L. Fialkow (cf. [2]) that if two invertible bilateral weighted shifts are quasisimilar, then they are similar. Thus it sufficies to show that no Rosenthal shift is similar to its adjoint.

Let $B_{w=\left\{w_{n}\right\}_{n \in \mathbb{Z}}}$ be any invertible bilateral shift. Recall (2): $B_{w}^{*} e_{n}=w_{n-1} e_{n-1}$, $n \in \mathbb{Z}$, and, making the same change of basis used in the proof of Theorem 1.3 (i.e., $f_{n}=e_{-n}, n \in \mathbb{Z}$, and then $\left.k=-(n+1)\right)$, one obtains easily that

$$
B_{w}^{*} f_{k}=w_{k}^{-1} f_{k+1}, \quad k \in \mathbb{Z} .
$$

(The purpose of this change of basis is to change $B_{w}^{*}$ from a backward shift to a forward shift, which is necessary before one can apply the known necessary and sufficient condition for similarity of two forward bilateral shifts.) According to $\left[6\right.$, p.60], in order that two invertible bilateral weighted shifts $B_{w_{1}}$ and $B_{w_{2}}$ with 
corresponding sequences $\beta^{(1)}$ and $\beta^{(2)}$ (via (4) and (5)) be similar it is necessary that there exist some $k_{0} \in \mathbb{Z}$ such that the sequence

$$
\left\{\frac{\beta_{k_{0}+n}^{(1)}}{\beta_{k_{0}}^{(1)} \beta_{n}^{(2)}}\right\}_{n \in \mathbb{Z}}
$$

is bounded above. Since we are discussing a Rosenthal shift and its adjoint (turned into a forward shift by (11)), with corresponding $\beta$ sequences $\left\{\beta_{n}\right\}$ and $\left\{\beta_{n}^{*}\right\}$ given by (1), (4), (7), and (11), we know that $\beta_{n}^{*}=\beta_{n}^{-1}, n \in \mathbb{Z}$, and therefore (12) becomes

$$
\left\{\frac{\beta_{k_{0}+n} \beta_{n}}{\beta_{k_{0}}}\right\}_{n \in \mathbb{Z}} .
$$

Since for $k_{0}$ fixed, $\beta_{k_{0}}^{-1}$ is a positive constant and $\beta_{j} \geq 1, j \in \mathbb{Z}$, for any Rosenthal shift, if the sequence $\left\{\beta_{n}\right\}_{n \in \mathbb{N}}$ is not bounded above, then the sequence in (13) is not bounded above, and the Rosenthal shift is not similar to its adjoint. Now choose $n=m^{2}+m, m \in \mathbb{N}$, and note from (6) and (7) that

$$
\lim _{m \rightarrow+\infty} \beta_{m^{2}+m}=\lim _{m \rightarrow+\infty} r_{m}^{m}=+\infty,
$$

so the proof is complete.

We complete this short exposition of Rosenthal shifts with the following result about transitivity.

Proposition 1.5. Every Rosenthal shift has a nontrivial hyperinvariant subspace.

Proof. According to Atzmon[1, Th.5.1, II], in order for an invertible bilateral weighted shift $B_{\left\{w_{n}\right\}}$ with corresponding sequence $\left\{\beta_{n}\right\}$ given by (4) and (5) to have a nontrivial hyperinvariant subspace, it sufficies to know that the series

$$
\sum_{n \in \mathbb{N}_{0}}\left(\ln \beta_{n}+\ln \beta_{-n}\right) /\left(1+n^{2}\right)
$$

is convergent. In our case, recall that (6) gives that the sequence $\left\{\beta_{n}\right\}$ is given by

$$
\beta_{n}=\beta_{-n}, \beta_{n^{2}}=1, \beta_{n^{2}+j}=r_{n}^{j}, \beta_{n^{2}+n+j}=r_{n}^{n+1-j}(1 \leq j \leq n), \quad n \in \mathbb{N},
$$

and therefore the series in (14) is equal to

$$
\sum_{n \in \mathbb{N}} \frac{2 \ln \beta_{n}}{n^{2}+1}=\sum_{n \in \mathbb{N}}\left[\sum_{j=1}^{n}\left(\frac{2 j \ln r_{n}}{\left(n^{2}+j\right)^{2}+1}+\frac{2(n+1-j) \ln r_{n}}{\left(n^{2}+n+j\right)^{2}+1}\right)\right]
$$

which is obviously dominated by

$$
\sum_{n \in \mathbb{N}} \sum_{j=1}^{n}\left(\frac{2 n \ln r_{n}}{\left(n^{2}\right)^{2}+1}+\frac{2 n \ln r_{n}}{\left(n^{2}\right)^{2}+1}\right)=\sum_{n \in \mathbb{N}} \frac{4 n^{2} \ln r_{n}}{n^{4}+1},
$$


which obviously converges since $\ln r_{n} \rightarrow 0$ by (6).

Acknowledgement. The authors express their gratitude to Ron Douglas and Ciprian Foias for useful chats about Rosenthal shifts. This research was supported by Kyungpook National University Research Fund, 2012.

Added in proof. After this paper was accepted for publication, Professor Aharon Atzmon kindly informed us that it results from his paper, The existence of translation invariant subspaces of symmetric self-adjoint sequence spaces on $\mathbb{Z}, \mathrm{J}$. Funct. Anal. 178(2000), 372-380, that a better result than Proposition 1.5 is true, namely: Every bilateral weighted shift $B_{\left\{w_{n}\right\}}$ with associated sequence $\left\{\beta_{n}\right\}_{n \in \mathbb{Z}}$ (via (4)) satisfying $\beta_{n}=\beta_{-n}, n \in \mathbb{Z}$, has a nontrivial hyperinvariant subspace.

\section{References}

[1] A. Atzmon, On the existence of hyperinvariant subspaces, J. Operator Theory 11(1984), 3-40.

[2] L. A. Fialkow, A note on quasisimilarility of operators, Acta Sci. Math. (Szeged) 39(1977), 67-85.

[3] H. Radjavi and P. Rosenthal, Invariant subspaces, Dover Publ. Inc. 2nd ed., 2002.

[4] H. Rosenthal, A weighted bilateral shift which is a possible counter-example to the hyperinvariant subspace problem, in preperation.

[5] H. Rosenthal, A new direction in classical harmonic analysis with applications to the hyperinvariant subspace problem, preprint.

[6] A. Shields, Weighted shift operators and analytic function theory, Topics Oper. Th., pp. 49-128. Math. Surveys, No. 13, Amer. Math. Soc., Providence, R. I., 1974. 